\title{
Are simulation and didactic crisis resource management (CRM) training synergistic?
}

\section{J B Cooper}

\section{Simulation may have an additive component to classroom style training, at least in the short term}

$\mathrm{F}$ ull environment simulation is achieving wide adoption despite weak evidence of its impact on outcome. It is doing so because it has strong face validity, creates much enthusiasm from both students and teachers, and because it is what other high hazard industries do to mitigate errors and to create and maintain a culture of safety. Yet most of us working with simulation technologies and techniques generally try to maintain our objectivity. We ask ourselves if it really does what we think it does, how much fidelity is needed to achieve our educational goals, and how we weigh the costs and benefits. When we are using it for non-technical training such as improving teamwork, we want to understand how it should be used to meet the real objective-creating real lasting behavior and culture changes that will make health care more effective and safer.

The paper in this issue of QSHC by Shapiro and colleagues ${ }^{1}$ demonstrates a model for using simulation to sustain behavior change and adds some additional evidence to bolster our general beliefs. But, as often happens with studies of educational and training interventions, we are left with many more questions than answers and are disappointed by an underpowered study, although not by much. That is not the fault of the investigators whose underlying methods were an advance over what we usually see in the world of non-technical simulation based training. The fault lies with having so few resources to perform the robust research designs needed, and also with the challenges of doing any research on human performance in naturalistic settings.

What is the utility of high fidelity, high realism, simulation based training for non-technical skills and culture change? We have plenty of evidence that those who experience it usually feel strongly that it is important for teaching skills which they do not other- wise experience or practice. ${ }^{2}{ }^{3}$ We have anecdotes illustrating how it appears to impact on clinical performance. ${ }^{4}$ Almost anyone who uses simulation to teach or reinforce teamwork or crisis resource management (CRM, or crew resource management as it is called in aviation) has encountered students who say they altered their fundamental way of doing things and working with their colleagues. I have heard many of these stories first hand, so I know the passion of those who have had such a transformational experience. That is one of the most useful applications of this form of simulation: transformational change for those who need first to recognize the problem before they can start to work on it. But fundamental, lasting, outcome altering organizational change cannot come with single interventions of one type. The important illustration from this study is how simulation can be coupled with other forms of CRM techniques to sustain improvements. Neither simulation nor non-simulation based training is likely to be effective alone for their intended purposes. Aviation, maritime, and nuclear industries all use combinations of stand-up training and simulation based training to establish and maintain human factors programs intended to minimize error, mitigate the error chain, and enhance performance. We get some tantalizing evidence in this new report that simulation has an additive component, at least in the short term, to classroom style training.

The general methodology used by Shapiro et al is illustrative of the kind of trials needed to produce evidence of transfer-of-training. The groups are randomized, there are sound validated measures of behavior with a measure of inter-rater reliability, and the raters are blinded to which cohort they are observing. But the study also demonstrates the flaws typical of educational studies: the sample size is too small, it is not linked to patient health outcomes (injury, death, reduced length of stay in hospital), there is no cost/benefit measure, and there are many subelements in the independent variable (degree of realism, quality of instruction, time of instruction, time between the MedTeams and simulation training) which can strongly impact on the effectiveness of training but are not examined in the experiment.

It is easy to criticize educational studies. I have not personally been involved with a successful one that is up to the standards of the "hard" sciences (which often themselves give us answers that later prove to be wrong by further research). This is difficult work but it needs to be done-even with the flaws-because each piece of evidence adds something to what we know. We also have to be willing to publish the negative results and to validate tools and approaches for studying simulation. ${ }^{56}$

Regardless of any criticism I might have of studies of simulation, when it comes to adopting simulation as an integral component of creating high reliability healthcare organizations, I accept and promote Gaba's observation that "... no industry in which human lives depend on the skilled performance of responsible operators has waited for unequivocal proof of the benefits of simulation before embracing it." ${ }^{\prime 7}$ Why should health care be different?

Qual Saf Health Care 2004;13:413-414. doi: 10.1136/qshc.2004.011544

Correspondence to: J B Cooper, Associate Professor of Anaesthesia, Harvard Medical School, Massachusetts General Hospital, Boston, Mass 021 14, USA; Director Biomedical Engineering, Partners Healthcare System Inc; Executive Director, Center for Medical Simulation; jcooper@partners.org

Competing interest disclaimer: The author is Executive Director of the center in which the simulation training described in this report was conducted but had no direct involvement in the study.

\section{REFERENCES}

1 Shapiro MJ, Morey JC, Small SD, et al. Simulation based teamwork training for emergency department staff: does it improve clinical team performance when added to an existing didactic teamwork curriculum? Qual Saf Health Care 2004;13:417-21.

2 Gaba DM, Howard SK, Fish K, et al. Simulation-based training in anesthesia crisis resource management (ACRM): a decade of experience. Simulation \& Gaming 2001;32:175-93.

3 Jha AK, Duncan BW, Bates DW. Simulator based training and patient safety. In: Making health care safer: a critical analysis of patient safety practices. Agency for Healthcare Research and Quality, US Dept of Health and Human Services, 2001:51 1-8.

4 Olympio MA. Simulation saves lives. Am Soc Anesthesiologists Newsletter 2001;65:15-9.

5 Gaba D, Howard SK, Flanagan B, et al. Assessment of clinical performance during 
simulated crises using both technical and behavioral ratings. Anesthesiology 1998;89:8-18.
6 Fletcher G, Flin R, McGeorge P, et al. Anaesthetists' non-technical skills (ANTS): evaluation of a behavioral marker system. Br J Anaesth 2003;90:580-8.
7 Gaba D. Improving anesthesiologists' performance by simulating reality (editorial). Anesthesiology 1992;76:491-4.
Informed consent

\section{Informed consent: don't throw out the moral baby with the critical bath water}

L Doyal

Informed consent is one of the most important bricks in the edifice of "right" medical treatment, but low standards must be recognised and corrected

$\mathrm{t}$

is well over two decades since Ian Kennedy published "Unmasking Medicine" based on his Reith lectures. He launched an inspired attack on medical paternalism in the UK which in many important respects has now been won-at least in principle. Clinical practice is now expected to embody the duty both to protect life and health and to respect individual autonomy-the right of competent patients to make informed choices about their medical options. Indeed, it is now both legally and professionally clear that, when these two duties are in conflict, the latter trumps the former. Ultimately, the competent adult patient has the final say about whether or not to accept or reject proposed treatment, even when refusing may mean death.

There is now a well articulated body of statute and case law designed to reinforce the right of patients to consent to or refuse treatment on the basis of appropriate information. Guidance from the GMC and professional organisations-particularly the BMA-does the same. ${ }^{2}$ The Department of Health has issued specific instruction about the standard of obtaining consent to which Trusts are expected to conform, including the structure and content of consent forms. ${ }^{4}$ In relation to medical education, courses abound in ethics and law applied to health care that emphasise the moral and legal importance of obtaining consent to a reasonable standard. Similarly, courses in communication skills are now offered to engender in young doctors the abilities required to meet this standard. Despite some continuing problems of organisational sensitivity-those of the sort highlighted in the Bristol Inquiry-we should not underestimate just how far the NHS has come with regard to respect for the autonomy of patients. ${ }^{5}$ This should be recognised and applauded.

\section{IS CONSENT TRULY INFORMED? Study by Habiba et al ${ }^{\circ}$}

In this issue of QSHC, however, a timely, interesting, and particularly well referenced paper by Habiba et al suggests the danger of too much optimism in this regard. ${ }^{6}$ The appearance of obtaining formal written consent may reflect a reality that is far removed from the moral goal of respect for individual autonomy. In a qualitative study based on a population of 25 women who had experienced either elective or emergency O\&G surgery, they conclude that the process of obtaining informed consent can become a ritualised formality that has little to do with either effective communication or even the confirmation of real choices when consent forms are signed. With regard to elective care, many of the women interviewed felt that that the process of obtaining consent often had little to do with the goals of educating them to make a truly informed choice. Further, other women receiving emergency care did not see the point of the consent process at all and sometimes did not refuse care when they said that they really wanted to. Among other things, the authors conclude that, if informed consent is going to live up to its moral ambitions, the complexity of the consent process should receive greater attention in conventional bioethics literature.

One of the most useful aspects of this study is its narrative methodology. In articulating it, the authors implicitly stress the importance of Trusts auditing the quality of consent obtained by staff and point out that, for this to be effective, it must include more than checking whether or not basic formalities of consent and signing consent forms are being observed. Institutional rituals of obtaining consent must not be confused with truly informed choice, and ways need to be found of monitoring the degree to which staff are achieving anything like the latter. The methodology of such audits should always include some element of triangulation through in depth discussion with a reasonable sample of patients about the quality of their experience.

\section{General view}

It is important, however, to place this particular research into a wider context. On the one hand, many discussions within the literature on consent also stress the importance of the quality of the learning processes of patients and of the relative insignificance of consent forms in this regard. For example, recent guidance from the Department of Health states that: "When a patient formally gives their consent to a particular intervention, this is only the end point of the consent process. It is helpful to see the whole process of information provision, discussion and decision-making as part of 'seeking consent'."4 (page 18) The GMC makes similar points in their guidance on consent, as do the BMA and others. Therefore, the question that is posed is why, in light of our understanding of these dangers, does this ritualisation of consent continue in so many settings?

The general answer is partly reflected in the moral maxim: "ought implies can". There is little point insisting that someone ought to do something in principle when they are incapable of doing so in practice. Clearly, as the authors suggest, the experience of the process of communication prior to the signing of consent forms should be a rich and textured one. However, for this to be achieved much remains to be done. Despite undergraduate courses in ethics, law and communication skills, the fact remains that many healthcare professionals have not had such learning experiences. Without a common denominator of basic skills, it is difficult to know what can reasonably be expected of staff.

Furthermore, against the background of a health service increasingly driven by targets, it has become commonplace that consultation time has fallen. Without sufficient time for an empathetic relationship to develop between clinician and patient, along with sufficient time to communicate even minimum 
amounts of appropriate information, it is hardly surprising that patients may sometimes feel that interactions are abrupt and lack meaning. This would be the case irrespective of the ethical and legal understanding and the communication skills of their clinicians. Of course, this problem may be mitigated by the effective use of published information about surgical and medical procedures. However, the development and design of such literature is not easy, especially regarding the communication of understandable information about risks. ${ }^{7}$ Effectiveness entails the consumption of scarce NHS resources. These may not be available because of the need to use what resources are available simply to optimise the quality and safety of clinical interventions. In short, good ethics costs money and the NHS has some way to go in creating hospital or primary care environments that are consistent with optimal success in obtaining truly informed consent.

\section{CONCLUSION}

It is difficult to know how representative the study by Habiba et al is about the quality of consent within O\&G. On the one hand, we know nothing of the communication training and skills of the staff who obtained consent from the women who were interviewed or the time and other resources that they had to do so. On the other hand, and this is always a problem with in depth interview techniques, it is hard compare a process that has engendered complaints with the level of complaints there would be if there were no such process at all, even a substandard one. The fact remains that the doctrine of informed consent is one of the most important bricks in the edifice of what Kennedy calls "right" medical treatment. ${ }^{8}$ Without it, patients would be little better than slaves to whom doctors could use their "clinical discretion" to treat as they pleased. We want the communication and negotiation associated with informed consent to be as effective as possible and to recognise and correct low standards where they exist. But we do not want to throw out the moral baby with the critical bath water when and if we discover it. Certainly, some of the women having elective O\&G care who were interviewed understood this. They indicated that having to sign a consent form reminded them that only they could ultimately determine whether or not they would receive treatment. Indeed!

Qual Saf Health Care 2004;13:414-415.

doi: 10.1136/qshc.2004.011858

Correspondence to: Professor L Doyal, Department of Medical Ethics, Queen Mary University of London, London El 2AD, UK; I.doyal@qmul.ac.uk

\section{REFERENCES}

1 Kennedy I. Unmasking medicine. London: Paladin, 1983.

2 General Medical Council. Seeking patients consent: the ethical considerations. London: GMC, 1998.

3 British Medical Association. Report of the consent working party. London: BMA, 2001

4 Department of Health. Good practice in consent implementation guide: consent to examination or treatment. London: Department of Health, 2001

5 Bristol Royal Infirmary Inquiry. Learning from Bristol: the report of the public inquiry into children's heart surgery at the Bristol Royal Infirmary 1984-1995, Bristol Royal Infirmary Inquiry, 2001

6 Habiba M, Jackson C, Akkad A, et al. Women's accounts of consenting to surgery: is consent a quality problem? Qual Saf Health Care 2004:13:422-7.

7 Doyal L. Informed consent: moral necessity or illusion? Qual Health Care 2001;10(Suppl I):i29-33.

8 Kennedy I. Treat me right. Oxford: Clarendon Press, 1988.

\section{The consultation game}

\section{G Elwyn}

\section{Exploring the use of game theory to address quality}

G ame theory and clinical practice seem an unlikely marriage and it may not be obvious how such a combination could improve the quality of clinical consultations. But this area of applied mathematics seems set to reduce some of our quintessential human foibles to a predictable set of behaviours based on what you win and what you lose (forget about how you play the game-at last it's officially all about winning). Game theory could be a sharp new tool for dissecting the mass of behaviours at play in the medical consultation, a historically paternalistic human interaction that stretches back as far as Hippocrates.

Medicine is a service delivered by a mix of episodic and repeated interactions between humans, medicated by the use of technologies such as tests, drugs and procedures. There is clear evidence that there is communication failure in consultations and that adherence to advice and treatment is $40-50 \%$ less than optimum, yet explanations and successful interventions to address this problem are rare. It is also clear from many empirical studies that the quality of the consultations and communication processes is far from optimum when examined from patient perspectives, and results in inefficiencies, errors and costs, not least the medicolegal expense of patient claims.

\section{PRINCIPLES OF GAME THEORY}

The central principle in game theory is that "players think about what others are likely to do, and do so with some degree of thought" ${ }^{1}$ Although there are many types of games used to explore this "thinking", all have a structure that involves an interaction leading to a reward or a loss between people, in pairs or, more recently, between and within groups. The "laboratory rat" of game theory is called the Prisoner's Dilemma where two players independently face a decision whether to collaborate or defect (box 1).

The Prisoner's Dilemma predicts a rational strategy for players, one of mutual defection (the Nash equilibrium) which gives a safe but low return over many interactions, rather than cooperation which offers a greater reward but at a greater risk. What is surprising is that humans spurn the rational strategy more often than not, preferring instead to cooperate. This outcome is one demonstrated repeatedly, not just in the Prisoner's Dilemma but in more complex games such as the Centipede.

How this irrational behaviour persists in the "survival of the fittest" paradigm is ironically best explained by those evolutionary theorists who have looked to game theory themselves, and suggests that our oversized cerebral cortex with its unique consciousness has arisen not from a "dog eat dog" strategy of survival but from a Machiavellian drive to forge allegiances and beat the competition using guile rather than brute force. $^{2}$

The problem of cooperation is an area of active research where interdisciplinary debates between game theoreticians (mathematicians generally), 


\section{Box 1 The Prisoners' Dilemma}

Two prisoners independently face a decision whether to collaborate or defect where their individual decisions, when considered together, lead to differing payoffs. The possibilities are mutual cooperation, mutual defection, or a situation where one has decided to cooperate and the other to defect. One-sided defection offers the largest reward to the defector and the least reward to the collaborator. Mutual cooperation offers equal rewards to both. Mutual defection also offers equal rewards to both but at a marginally lower level and, according to the theory, this is the rational strategythe so-called Nash equilibrium, after the mathematician John Nash.

economists, psychologists, and social scientists are raging. Those disciplines accommodating broader perspectives, such as psychology, argue that human interaction is complex. Decisions about payoffs, they contend, are influenced as much by personal and contextual variables as they are by simplistic mathematical type assessment of gain or loss by interacting with others. Names such as "behavioural"1 and "psychological" game theory ${ }^{3}$ signal the ongoing discussions.

\section{APPLICATION OF GAME THEORY TO MEDICINE}

Game theory could help us to understand and explain the extent of cooperation and defection in medical interactions. As a profession, we have been slow to look beyond the boundaries of medicine to other disciplines and, because of this conservatism, research into medical processes has neglected game theory as a potential theoretical framework for analytical work.

Other disciplines have been quicker to see the potential of game theory and have used it extensively to examine many areas of human life. Axelrod in particular has applied the theory in innovative ways to consider sociological issues and to examine policy implications. ${ }^{4}$ Gutek examined the differences between commercial service encounters (one-off consultations) and service relationships (repeated consultations) in many walks of life such as hairdressing, financial advice, and family physicians, and found disadvantages to both consumer and provider in each design. ${ }^{5}$ Medicine could usefully extend this work to examine where continuity adds (or detracts) value to professionalpatient interactions.

Existing research on the medical consultation is devoid of a theoretical basis. While there are plenty of prescriptive models such as the "patient centred method", the "Cambridge Calgary" approach ${ }^{7}$ and so on, this field does not seem to have provided a theory on which to examine the empirical findings. Descriptive work such as that by Byrne and Long, ${ }^{8}$ assessment of videotapes in the Royal College of General Practitioners' examination," and the recent analyses by discourse analysts reveal that practitioners do not come anywhere near performing according to the prescribed "idealised" models. $^{10}$

So where does the fault lie? With the practitioners or with the models? The models are based on professionally constructed idealised communication methods and have not been based on adequate theories of how humans interact, given the differing payoffs for patients and practitioners. For health professionals, keeping to time and limiting their exposure to large volumes of patients is a way of managing their workload, maximising income, and keeping sane. At the same time they need to retain the cooperation of patients by building trust, obtaining information, and proving to be good at solving and dealing with problems. The payoffs for doctors of engaging in advocated methods of communicating-exploring ideas, concerns and expectations, for instance-are not so clear. Payoffs for patients are different in different situations. Fast provision of a sought-for medication is all some people are looking for; others are looking for supportive ongoing relationships where chronic illnesses are explained and mastered in partnerships, while others are looking for a screening investigation but are unaware of the possible harms involved, and so on. It may well be time to pause for thought and to examine the motivations for doctors and patients in a different way.

When combined with developments in neuroscience, particularly real time MRI scans, games such as the Prisoner's Dilemma show that cooperation leads to activation of brain areas associated with reward processing and learning, reinforcing the behaviour over and above the payoff itself. ${ }^{12}$ So it appears that humans are programmed to be more than calculating machines, interested only in maximising utility, as pure game theory would have it. Instead, we like to work together, to some extent at least, and it is a matter for further research to explain how. Game theory offers medicine a potential method, yet the complexity and the layered nature of the consultation cannot be easily modelled by one or other of the existing games in the game theory playroom. In their paper in this issue of QSHC, Tarrant and colleagues ${ }^{11}$ concede that this will be a considerable challenge. If it is possible, perhaps we can then think about improving the quality of the consultation process and create gains for practitioners, patients, and health service economists.

\section{ACKNOWLEDGEMENTS}

The author thanks Jenny Kowalczuk for contributing to the drafting of this commentary.

Qual Saf Health Care 2004;13:415-416. doi: 10.1136/qshc.2004.011999

Correspondence to: Professor G Elwyn, Primary Care Group, University of Wales Swansea, Swansea SA2 8PP, UK; g.elwyn@swansea.ac. uk

\section{REFERENCES}

1 Camerer CF. Behavioural game theory. New York: Russel Sage Foundation, 2003

2 Barkow JH, Cosmides L, Tooby J, eds. The adapted mind. New York: Oxford University Press, 1992.

3 Colman AM. Cooperation, psychological game theory, and limitations of rationality in interaction Behav Brain Sci 2003;26:139-98.

4 Axelrod R. The complexity of cooperation. Agentbased models of competition and collaboration. Princeton: Princeton University Press, 1997.

5 Gutek BA, Groth M. Distinguishing between service relationships and encounters. J App Psychol 1999;82:218-33.

6 Stewart M, Brown JB, Weston WW, et al. Patient centred medicine: transforming the clinical method. Thousand Oaks, CA: Sage Publications, 1995.

7 Silverman J, Draper J, Kurtz SM. The CalgaryCambridge approach to communication skills teaching. 1: Agenda-led and outcome based analysis of the consultation. Educ Gen Pract 1996;7:287-99.

8 Byrne PS, Long BEL. Doctors talking to patients. London: HMSO, 1976.

9 Campion P, Foulkes J, Neighbour R, et al. Patient centredness in the MRCGP video examination: analysis of large cohort. BMJ 2002;325:691-2.

10 Gwyn R, Elwyn G, Edwards A, et al. The problematic of decision-sharing: deconstructing 'cholesterol' in a clinical encounter. Health Expect 2003;6:242-54

11 Tarrant C, Stokes T, Colman AM. Models of the medical consultation: opportunities and limitations of a game theory perspective. Qual Saf Health Care 2004;13:461-6.

12 Anon. Why we do what we do. New Scientist 2004; 183:32-6. 